\title{
Transition to adult life for students who are Black and have disabilities: What do we know and what do we need to know?
}

\author{
Colleen A. Thoma ${ }^{\mathrm{a}, *}$, Martin Agran ${ }^{\mathrm{b}}$ and LaRon A. Scott ${ }^{\mathrm{a}}$ \\ ${ }^{a}$ Department of Counseling and Special Education, Virginia Commonwealth University, Richmond, VA, USA \\ ${ }^{\mathrm{b}}$ University of Wyoming, Laramie, WY, USA
}

Revised/Accepted March 2015

\begin{abstract}
.
BACKGROUND: Although there is considerable research that has identified effective strategies to support the transition from school to adult life for young adults with disabilities, post-school outcomes for culturally linguistically or economically diverse (CLED) students with disabilities, particularly students who are Black and have disabilities, remain abysmal.

OBJECTIVE: This article reviews the literature on transition practices for students who are Black and have disabilities to determine what is known about their unique challenges, as well as what can be done to improve their postschool outcomes. METHODS: The current published literature was reviewed to identify articles that examined the impact of transition practices on postschool outcomes for students who are Black and have disabilities.

RESULTS: Although the research literature on students who are Black and have disabilities in transition programs is limited, a number of practices were identified as improving transition planning and services for these students, including several that enhance student self-determination in the process.

CONCLUSIONS: As the postschool outcomes for this group of youth students who are Black and have disabilities continues to be abysmal, this review of the literature revealed that there is a need to conduct research to further identify barriers and effective practices needed to overcome them. Implications for practice as well as further research are discussed.
\end{abstract}

Keywords: Transition, youth with disabilities, cultural diversity, culturally responsive practices, Black youth with disabilities, post-school outcomes

\section{Introduction}

While transition outcomes for all students with disabilities continue to lag behind outcomes of students without disabilities, evidence reflects that outcomes for students with disabilities from culturally, linguistically, and economically diverse (CLED)

\footnotetext{
*Address for correspondence: Colleen A. Thoma, Virginia Commonwealth University, Department of Counseling and Special Education, 1015 W. Main Street, P.O. Box 842020, Richmond, VA 23284-2020, USA. Tel.: +1 804827 2651; Fax: +1 804225 3554; E-mail: cathoma@vcu.edu.
}

backgrounds - particularly, students who are Black and have disabilities - lag even further behind. For example, the National Longitudinal Transition Study2 (Sanford et al., [NLTS-2] 2011) found students who are Black and have disabilities less likely to be employed after high school. Also, although $86 \%$ of surveyed students who are Black with disabilities have been employed, they most likely worked as service providers or in cleaning and maintenance, had worked fewer hours per week compared to coworkers who are White, and were least likely to receive any benefits (e.g., sick leave, paid vacation, 
retirement benefits) (Sanford et al., 2011). Also, they were least likely to report that they liked their jobs very much and most likely to report that they did not like their jobs much.

This group of individuals with disabilities lags behind in other postschool outcomes as well. Youth who are Black with disabilities are less likely to transition to postsecondary education; only $21 \%$ of youth who are Black with disabilities go on for postsecondary education compared to $35 \%$ of those who are White (Wagner et al., 2005). Further, they were least likely to report that they lived independently and were most likely to be parents out of wedlock, with only $58 \%$ reported satisfaction with their living condition. Additionally, they least likely visited friends outside school or work. They most likely reported negative community involvement, or having been (a) stopped by police for something other than a traffic violation, (b) had been incarcerated, or (c) were on probation or parole. Ultimately, they reported the lowest rates of maintaining a savings or checking account or using a credit card, which are financial independence indicators and provide evidence that Black students are most likely to live in poverty (Cameto et al., 2004; Wagner et al., 2005).

These poor outcomes for students who are Black and have disabilities come at a time when the population of the United States is becoming increasingly diverse. According to the U.S. Department of Education, $45 \%$ of all students in public schools are considered to be CLED, or more specifically categorized by the federal government as racially, ethnically, and linguistically diverse (Aud et al., 2011). This increase in the population of students who are CLED has resulted in the need for public schools to improve their ability to use culturally responsive practices to respond to and meet their needs. In particular, these efforts need to include culturally responsive and inclusive educational services (Hughes et al., 2013). First, the field must address issues of disproportionality or over-representation of CLED students in special education (Ford, 2012). Students who are Black are more likely to be referred to and found eligible for special education services as compared to White or Asian children and youth (National Research Council, 2002), particularly those students from high poverty areas (Hughes et al., 2013). In addition, while students receiving special education services are more likely to drop out of high school in general, this impacts youth who are Black with disabilities in particular. While $62.5 \%$ of White youth with disabilities earn their high school diploma, only
$39.2 \%$ of students who are Black with disabilities do so (U.S. General Accounting Office, 2003). Yet, as Ford (2012) points out, there is little research to guide the field to improve these outcomes and research is needed to differentiate between strategies that work for all students with disabilities and those that are effective for specific sub-populations of children and youth with disabilities and their families (e.g., students who are Black).

One such area in special education that warrants further scrutiny are the educational supports and services used to improve transition outcomes. The National Secondary Education and Transition Technical Assistance Center (NSTTAC; 2010) conducted a review of literature to identify evidence-based practices for transition planning, education and services and organized those practices following the components of Kohler's taxonomy for transition planning (1996). They identified 64 instructional practices that could be considered as evidenced-based, which were grouped as follows into the components of the taxonomy: a) student-focused planning (6 practices), student development (57), family involvement (1), and program structure (3). One taxonomy component required by IDEA, interagency collaboration, lacked any research to guide its implementation.

This review of evidence-based practices for transition also found that there is a need for research that focuses on specific sub-populations of students and to include that information in the findings (Trainor, 2008). Without this disaggregated information, it is difficult to determine whether these evidence-based practices are effective in improving postschool outcomes for students who are Black and have disabilities or whether different approaches are needed to address differing challenges for this population. In particular, there is widespread agreement that self-determination represents a critical set of skills necessary to youth transitioning to adulthood (Storey \& Miner, 2011; Wehman, 2011) and is a key component of high-quality transition services (Cobb, 2009). High student self-determination has been linked to in-school academic gains (Stodden \& Dowrick, 2001) and improved post-school employment (Wehmeyer \& Schwartz, 1998), post-secondary education (Raskind et al., 1999; Thoma \& Getzel, 2005), and positive community living outcomes (Wehmeyer \& Schalock, 2001). In particular, the positive relationships between self-determination and transition outcomes as reported in the research literature prompted the Council for Exceptional Children's Division on Career Development and Transition to 
call for the inclusion of self-determination instructional strategies in transition programs to prepare students for their transition from high school to community working and living (Field et al., 1998). Raskind et al. (1999) indicated that teaching disability awareness, goal setting, and other self-determination skills need to become an essential part of secondary special education curriculum.

Research has shown that promoting selfdetermination for students with disabilities can have a positive lifelong effect, with self-determined adults with disabilities more likely to have a better quality of life (German et al., 2000; Martin et al., 2003; Raskind et al., 1999; Wehmeyer \& Schwartz, 1998; Wehmeyer \& Schalock, 2001). In response, many instructional materials, programs, and strategies have been developed over the last two decades to assist teachers and other service providers in incorporating self-determination instruction into their curriculum (Field \& Hoffman, 1994; Halpern et al., 1995; Hughes \& Carter, 2000; Martin \& Marshall, 1996; Wehmeyer, Agran, \& Sands, 1998; Wehmeyer \& Sands, 1998; Thoma, 1999). Yet despite this research, there are some experts who question whether self-determination is relevant for youth who are CLED and, in particular, students who are Black and have disabilities. Kalyunpur and Harry (2012) argue that self-determination may be an egocentric (self-driven) concept that does not necessarily fit for some individuals with disabilities and their families who have a socio-centric (communal) orientation and a goal of interdependence rather than independence (such as those from Black or Latino heritage). They further link the concept of self-determination to such Anglo-American ideals as independence and the right to maximize one's own potential, which they describe as being in conflict with cultures that believe in "hierarchical interdependence, whereby people with disabilities are not stigmatized because they are dependent because, in a way, everybody is dependent on each other and everybody has a social role" (p.108). In a review of literature about self-determination for students with disabilities from CLED backgrounds, Shogren (2011) found that, although some of the research on the impact of self-determination on post-school outcomes included students from CLED backgrounds, their numbers were small and comparison data analysis across or among groups was not done. However, in contrast to Kalyunpur and Harry (2012), Shogren (2011) suggested that self-determination does have "universal value if individualized to consider each student's unique situation in terms of cultural and systems-level variables" (p. 115). This difference in perspective highlights the need to examine whether self-determination has a different impact on transition outcomes of specific sub-groups, or whether different strategies are needed to support the development and use of self-determination skills in transition planning for students from specific CLED backgrounds, including students who are Black and have disabilities. For this group of students in particular who experience some of the worst transition outcomes, a better understanding of the role of self-determination and other evidence-based practices for improving transition outcomes is critically needed.

Additionally, whether or not self-determination is perceived by stakeholders to be valued or not, it remains uncertain whether students who are Black and have disabilities are being provided selfdetermination instruction. Washington, Hughes, and Cosgriff (2012) conducted a study to examine this question and found that students who were Black, with and without disabilities, were significantly less likely to receive instruction on self-determination, less likely to use self-determination strategies (e.g., choice making, self-evaluation), or have an active role in educational planning or leading their IEP meetings. Similarly, in a study conducted by Hughes et al. (2013), which involved 47 participants, the majority of whom were Black, most of the students who were served in self-contained programs were not taught or used self-determination skills. In contrast, students in general education settings used self-determination skills at a higher level than students in self-contained programs but still at low levels. Given the positive outcomes associated with self-determination intervention for the general population of students with disabilities, we feel that further research is needed to determine its effects or differences with specific populations.

The purpose of this review of literature is to examine transition practices used with students who are Black and have disabilities to improve their postschool outcomes. The specific questions addressed were: a) to what extent have evidencebased practices for transition been used for students who are Black and have disabilities to support their transition from school to adult life; b) to what extent do these practices support the transition from school to adult life; and c) to what extent have these youth and their families been involved in transition planning? 


\section{Methods}

\subsection{Information retrieval}

A search of PsychoINFO, ERIC, and Academic Search Complete databases was conducted to identify studies and articles published between January 1990 and January 2013 that explored transition practices and their impacts on improving the post-school outcomes of students who are Black and have disabilities. The year 1990 was chosen as it was the first year that the Individuals with Disabilities Education Act (IDEA; PL 101-476) mandated transition planning and services for all youth with disabilities. Search terms included: transition, special education, self-determination, cultural diversity, African American, and Black. Searches were conducted using all possible combinations and sequences of these terms. In addition to the electronic databases, the reference lists of included articles were reviewed to identify potentially relevant research.

Once articles were identified using the search criteria, their abstracts were reviewed using inclusionary criteria. This included that articles were: a) published in a peer-reviewed journal; b) focused on schoolprovided supports and services for transition-aged youth (i.e., students aged 16 or above); c) quantitative, qualitative, or mixed methods research studies; and d) included disaggregated information about transition for students who were Black with disabilities. Articles excluded from this review of literature were policy or opinion statements, program descriptions without the collection of data to determine the effectiveness or impact of the supports and/or services provided, or included youth who were Black with disabilities (or their parents) without an ability to understand how the intervention impacted their transition outcomes.

\subsection{Analysis of literature}

From more than 100 articles identified from the initial search of literature, only six articles were identifying as meeting the inclusionary criteria. A majority of the articles excluded from this review did not include youth who were Black with disabilities or were theoretical or conceptual perspectives that did not include any data collection or analysis. Five articles in the original group of articles included students who were Black with disabilities or their parents as participants in the study, but did not disaggregate their findings. This made it impossible to determine findings specific to youth who were Black and had disabilities, the focus of this review. Content of included articles were examined to determine: a) purpose; b) research design; c) participants and basic demographic information; d) dependent and independent variables; e) transition practices; f) results; and student and parent involvement.

\subsection{Coding}

Once identified for inclusion, articles were analyzed for the transition strategies used and/or recommended as well as components described consistent with parent and student involvement. Barriers to the use of effective transition and student and parent involvement were coded according to Kohler's (1996) taxonomy. Key findings from each article were summarized in a table to assist with an analysis of existing research, as well the identification of emergent themes. The first author took the lead in conducting the review of literature, based on the framework identified by co-authors related to evidence-based transition planning and services, and student and family involvement.

\subsection{Reliability}

Reliability data on content information were obtained from all six (100\%) studies. One researcher was primarily responsible for conducting the literature review, applying the inclusionary criteria to identify the six articles, and then coding each of the articles. Inter-observer reliability data were then gathered by having another researcher review the articles and independently coding them. Reliability was calculated by dividing the number of agreements in coding by the total number of total items coded to determine a percentage of agreement. Overall inter-observer reliability ranged from $91.5 \%$ to $100 \%$ agreement with a mean of $94.8 \%$ agreement. For items coded differently, the two researchers discussed their coding differences and described their rationale for the original code. Discussion resulted in agreement on both codes and those changes were made in the findings reported.

\section{Findings}

Of the six articles, two used quantitative methods (Geenen, Powers, \& Lopez-Vasquez, 2001; Hasnain \& Balcazar, 2009), one used qualitative (Goff, 
Martin, \& Thomas, 2007), and the other three used mixed methods (Geenen et al., 2003; Landmark, Zhang, \& Montoya, 2007; Leake, Burgstahler, \& Izzo, 2011). None of the six articles involved the investigation of a specific intervention designed to improve transition outcomes; rather, they were focused on describing or defining current practice. Two studies included youth with disabilities as participants, seeking their perspectives about factors that impact their transition goals and outcomes (Goff et al., 2007; Leake et al., 2011), while three studies included parents and/or special educators as participants (Geenen et al., 2001, 2003; Landmark et al., 2007). The sixth study was a secondary analysis of data collected from the NHIS-D longitudinal study to determine predictors of community-based versus facility-based employment outcomes for individuals with disabilities (Hasnain \& Balcazar, 2009). The following section describes in more detail the six selected articles.

\subsection{Extent to which evidence-based practices are used to support transition planning and services}

Only one article included in this review of the literature focused solely on the transition of youth who were Black and had disabilities (Goff et al., 2007). All other articles focused on youth from culturally and/or linguistically diverse backgrounds and their families, which included those who are Black.

Goff et al. (2007) examined experiences of a group of youth who were Black, identified as atrisk for school failure, and attended an alternative middle school. Their purpose focused on determining the extent to which Fordham and Ogbu's (1986) controversial burden of acting White impacted their educational and transition experiences as well as their postschool goals and expectations. The burden of "acting White" describes a phenomenon whereby Black youth equate academic achievement with "acting White" and are alienated or ridiculed from their Black peers when they did so (Fordham \& Ogbu, 1986). Each student acknowledged that he or she experiencing the burden of "acting White," which their teachers corroborated. Goff et al. (2007) also found that although teachers did not describe any strategies that could be used to intervene, students who had clearly identified future goals and role models who were Black (including parents) were best able to "navigate the burden of acting White" (p. 138). Researchers concluded that students who are Black need skills in goal-setting, student-focused planning, and increased parent involvement in transition planning in a manner that is more culturally responsive that is, not just an imitation of mainstream culture.

Hasnain and Balcazar (2009) conducted a secondary analysis of transition outcome data collected through the 1994-1995 National Health Interview Survey on Disability (NHIS-D). Their survey did not address specific aspects of self-determination relative to the transition to employment, yet revealed significant differences in employment outcomes for youth who were Black and had disabilities compared to youth who were White and had disabilities. For example, youth who were Black were more likely live in poverty and be unemployed. Those employed more likely used informal supports to find employment rather than formal supports such as Vocational Rehabilitation Services. Additionally, they were more likely to be placed in facility-based employment settings and less likely to be placed in postsecondary education or community employment. The study highlighted the need to involve parents and other informal supports in the transition planning process and the need for an expanded understanding of parent involvement and interagency collaboration. Hasnain and Balcazar identified the "lack of connection between the racial/ethnic culture of Black adults with disabilities and the formal systems of support whose values reflect the mainstream, White, middleclass American culture" (p. 186).

Leake et al. (2011) conducted telephone surveys and focus group interviews with young adults with disabilities from CLED backgrounds to determine the impact of having a mentor when navigating the transition to adult life. Fifty-three percent (53\%) of youth with who were Black and had disabilities reported having had a mentor during high school and that they preferred having a mentor who had the same ethnic/racial background and disability. Participants reported that mentors served as a source of emotional and educational support, assisted with goal setting and problem solving, and provided assistance in negotiating service systems. As a consequence of these experiences, participants also noted their desire now to serve as a mentor to others (leadership). In terms of Kohler's (1996) taxonomy for transition, this study linked mentor support to: student-focused planning, student development of self-determination skills such as goal-setting, problem-solving, and leadership skill development, and interagency collaboration to negotiate service systems. Youth who were Black and had disabilities participating in the 
study also reported that having a mentor who shared their ethnic/racial background helped another area of student development-understanding one's cultural heritage and/or values.

\subsection{Strategies used to increase student and parent involvement in transition planning}

Transition planning typically focuses on the development of individualized education plans (IEPs) that identify goals to support students' transitions from school to postschool activities. Parent involvement in the IEP process has been required since the early years of U.S. special education policy, beginning with the 1975's Education for All Handicapped Children Act (P.L. 94-142). Thus, it is not surprising that parent involvement in transition planning would receive attention in research studies.

Geenen et al. (2001) conducted surveys of parents and school personnel to gain an understanding of the multicultural aspects of parent involvement in transition planning. They surveyed 308 parents of African American, Hispanic American, Native American, and European American descent and 52 school personnel. They found parents of AfricanAmerican descent believe that in terms of student development, it was important to engage their children in discussions about life after high school (goal setting/self-advocacy), using transportation services (independence), and self-caring for their disability (self-knowledge/self-awareness). They also reported that they believe it is important to teach their children cultural and family values. This aspect of transition education was stressed by parents while school personnel did not rate it as high a priority. Also, they reported being more likely to be involved in transition-related planning at home than in schoolorganized activities. Geenen et al. found that while parents of youth who were Black and had disabilities were actively involved in helping their children set goals, school personnel reported that they were not as involved as other parents.

Geenen et al. (2003) conducted both focus group and individual interviews with parents of youth with disabilities from CLED backgrounds to better understand their transition planning involvement. These parents reported a number of barriers to their involvement, including "power imbalance, psychological/attitudinal; logistic; information; communication; SES/contextual barriers and cultural factors of influences" (p. 8). Parents reported that the educational and transition needs of their chil- dren were misunderstood due to cultural background differences between themselves and school personnel. In addition, some parents reported that they were treated poorly because of their culture. Recommendations for increasing the transition planning involvement of parents of students with disabilities from CLED backgrounds include using parental advocates, providing information about the transition planning process to empower parents, and using flexible meeting formats that encourage parent participation.

Further, when the results were compared and used to identify focus group questions, the study revealed that youth with disabilities from CLED backgrounds faced greater barriers to transition than youth who were White and had disabilities. In particular, this study indicated that parents of youth with disabilities from CLED backgrounds believed that school personnel did not understand that their cultural background required different approaches to transition planning. Parents of youth with disabilities from CLED backgrounds indicated that they used a range of informal family and community supports not addressed by the typical school-based transition planning process. In addition, they reported that although they wanted their sons and daughters to be more selfsufficient, they did not necessarily equate that with living independently.

Landmark et al. (2007) also examined the transition experiences of parents of youth with disabilities from CLED backgrounds. They conducted telephone interviews and analyzed those transcripts using both quantitative and qualitative methods. Their study found that parents reported that while they did not know about school-based transition processes they were able to identify their child's strengths and weaknesses as well as their employment and adult life goals. Most reported that they attended transition meetings, although they did not necessarily understand the legal requirements for those meetings. They were more likely to report that participation in parent transition planning included assisting their children at home and in the community more so than parents of youth who were White and had disabilities who indicated that parent transition planning participation centered on school-based meetings. Parents of youth who were Black and had disabilities indicated that supports designed to improve transition services should focus on community and agency support for their children as well as financial support to allow them to be more involved in the process. 


\section{Synthesis of key themes across articles}

Several key themes were identified in the review. These included: Need for Clear Transition Goals, Available Resources, Link to Shogren's Findings, and Future Research Needs. A discussion of each follows.

\subsection{Need for clear transition goals}

The pool of reviewed articles was admittedly limited, nevertheless, an emergent theme identified was the lack of a consistent understanding of the goals for transition planning for students who were Black and have disabilities, their parents, and school personnel. This was particularly clear from the studies that examined strategies designed to increase parent transition planning involvement, as there was a discrepancy between the roles parents of youth who were Black assumed during the process and those school personnel wanted them to assume (Geenen et al., 2001, 2003; Landmark et al., 2008). Many of these parents reported that they wanted their sons and daughters to be more self-sufficient, learn to take care of themselves, be employed, and go to college (Geenen et al., 2001, 2003; Landmark, et al., 2008). However, school personnel more likely considered parent involvement relative to IEP meeting attendance, while parents of youth who were Black faced a number of barriers to attending these meetings and more likely valued things they did at home or in the community to support transition outcomes (Landmark, et al., 2008).

\subsection{Need to expand resources available to students who are black and have disabilities and their families}

A second emergent theme was the need for additional resources to improve transition outcomes for youth who were Black and had disabilities, for they are more likely to live in poverty, receive foster care services, be recipients of racial discrimination (Geenen et al., 2001, 2003; Landmark et al., 2007), and face additional challenges such as the burden of acting White (Goff et al., 2007). This requires transition planning that involves multiple service systems and strategies not typically included in school-based transition planning meetings, or self-determination transition curriculum. Researchers recommend a variety of resources including case managers, or mentors available to help students throughout the year in their communities (including summer) (Leake et al., 2011), the use of parent and/or student advocates to explain various systems and community resources (Geenen, 2001; Landmark et al., 2007), and expanding upon individuals invited to transition planning meetings to include agency staff currently working with the student, not just the adult service staff who might provide services in the future (Hasnain et al., 2009; Geenen et al., 2003).

\subsection{Link to Shogren's (2011) self-determination findings}

Shogren's (2011) review of literature on the self-determination of youth with disabilities from CLED backgrounds resulted in four themes: (a) "self-determined behavior can and does vary across cultural identities, (b) self-determination as operationalized in practice is often not culturally appropriate, (c) research must include voices and practices of diverse students and their families, and (d) change in multiple systems is needed" (p. 122). The present review of literature on transition for youth who were Black and had disabilities and their families support Shogren's findings. Studies included found these students more likely to set future goals, which typically differed from those school personnel considered appropriate. Also, these goals did not appear reflective of mainstream and cultural heritage. Geenen et al.'s studies $(2001 ; 2003)$ on parent involvement in transition highlighted a need to include instruction about and support for the family's cultural heritage and value system when transition planning. However, an individual's future goals are based on multiple influences, of which cultural heritage is one (Landmark et al., 2007). This requires a highly individualized system of support and in-depth training and expertise in both culturally responsive pedagogy (CRP) (Landmark et al., 2008) and the facilitation of student self-determination (Goff et al., 2007; Shogren, 2011). Transition education and services must address goals students set for their adult lives, rather than goals set by professionals (Hasnain \& Balcazar, 2009; Geenen et al., 2003; Shogren, 2011).

\subsection{Implications for future research and practice}

As indicated previously, an obvious limitation of this review was the limited number of articles examined and our failure to investigate the many other 
factors affecting the transition outcomes of youth who are Black and have disabilities. Nevertheless, we believe that self-determination represents a curricular area and skill set that may greatly enhance a student's future employability. Kohler (1996) and Shogren (2011) underscored the importance of this. The fact that relatively little has been written about how to promote the self-determination of youth who are Black and have disabilities and that their transition outcomes are so unacceptable begs for more research. While this review provided information about barriers to student self-determination and transition planning with parents, few studies were found providing specific guidance about a range of strategies to support the self-determination of and transition planning with youth who are Black and have disabilities (Balcazar et al., 2012; Leake et al., 2011). Future research must include the voices, perspectives, and input of these students and their families, as well as longitudinally investigate strategic impacts for improving these students' transition outcomes.

In addition to conducting research investigating impacts of self-determined transition planning on outcomes for youth who are Black and have disabilities, there is a need to clearly and concisely identify what it means to support student self-determination. While many studies have examined transition planning, interestingly, many did not identify whether they facilitated self-determination through the process and many of these described interventions generally focused on students' performance on one or more core component self-determination skills, such as problem-solving, choice-making, goal setting, or self-efficacy. Many failed to sufficiently address the need to support student resiliency, which can be conceptualized as a form of self-determination whereby individuals work toward meeting a goal despite external challenges and situations by identifying supports needed to accomplish a goal that might be outside typical family and school support networks.

As a number of researchers pointed out, youth who are Black and have disabilities may receive supports from agencies not typically invited to schools' transition planning processes and may be unfamiliar with strategies from special education designed to support student self-determination. Not only do we need to be sure that these supports are part of the transition planning process, research needs to clearly identify self-determination strategies used to help students achieve post-school goals so that self-determination impacts of can be determined. Likewise, the litera- ture reviewed for this study did not clearly identify CRP practices used as part of their interventions, so the goal of achieving a clearer understanding of which CRP practices improve our ability to support the transition from school to adult life for youth who are Black and have disabilities was not met. The perspectives/theoretical articles provided broad recommendations for transition practices that combined self-determination and CRP to improve transition outcomes, but few of the empirical studies comprehensively and clearly combined both.

\section{Conclusion}

It is ironical, if not unfortunate, that although self-determination is a required curricular need and planning approach for transition-age youth, its value for and applications to youth who are Black and have disabilities remain uncertain and limited. A functional relationship between self-determination and desired post-school outcomes for these youth has yet to be established. Interestingly, self-determination has not been generally accepted as a culturally valid practice (Kalyanpur \& Harry, 2012), and many transition programs continue not to include self-determination instruction in their transition programs, especially for youth who are Black living in high poverty schools. For example, Washington et al. (2012) examined the involvement in educational planning and use of self-determination strategies reported by students attending high-poverty schools predominately filled with students who were Black. Their findings revealed that these students had few opportunities to choose, solve problems independently, or learn self-determination skills. Given the growing literature that has validated and supported the value of self-determination in contributing to the realization of successful outcomes for young adults with disabilities, it is regrettable that the field has yet to understand the value of and how to instruct youth who are Black and have disabilities to become more self-determined. We trust that this paper will spur more interest in advancing this initiative, and, in doing so, help youth who are Black and have disabilities achieve the positive transition outcomes they and their parents want them to achieve.

\section{Conflict of interest}

The author have no conflict of interest to report. 


\section{References}

Aud, S., Hussar, W., Kena, G., Bianco, K., Frolich, L., Kemp, J.,\&...Hannes, G. (2011, May). The condition of education 2011 (NCES 2011-033). Washington, DC: U.S. Department of Education, National Center for Education Statistics. Retrieved from http://nces.ed.gov/pubs2011/2011033.pdf

Cameto, R., Levine, P., \& Wagner, M. (2004). Transition planning for students with disabilities: A special topic report of the findings from the National Longitudinal Transition Study2. Retrieved from www.nlts2.org/reports/2004_11/nlts2_ report_2004_11_complete.pdf

Cobb, B., Lehmann, J., Newman-Gonchar R., \& Alwell, M. (2009). Self-determination for students with disabilities: A narrative metasynthesis. Career Development for Exceptional Individuals, 32, 108-114. doi: 10.1177/0885728809336654

Education for All Handicapped Children Act of 1975, Pub. L. No 94-142, 20 U.S.C. SS 1401 et seq.

Field, S., \& Hoffman, A. (1994). Development of a model for self-determination. Career Development for Exceptional Individuals, 17, 159-169.

Field, S., Martin, J., Miller, R., Ward, M., \& Wehmeyer, M. (1998). A practical guide to teaching self-determination. Reston, VA: Council for Exceptional Children.

Ford, D. Y. (2012). Culturally different students in special education: Looking backward to move forward. Exceptional Children, 78, 391-405.

Fordham, S., \& Ogbu, J. U. (1986). Black students' school success: Coping with the "burden of acting White." Urban Review, 18, 176-206.

Geenen, S., Powers, L. E., \& Lopez-Vasquez, A. (2001). Multicultural aspects of parent involvement in transition planning. Exceptional Children, 67, 265-282,

Geenen, S., Powers, L., Vasquez, A. L., \& Bersani, H. (2003). Understanding and promoting the transition of minority adolescents. Career Development for Exceptional Individuals, 26, 27-46.

German, S. L., Martin, J. E., Marshall, L. H., \& Sale, R. P. (2000). Promoting self-determination: Using "Take Action" to teach goal attainment. Career Development for Exceptional Individuals, 23, 27-38.

Goff, C., Martin, J. E., \& Thomas, M. K. (2007). The burden of acting white: Implications for transition. Career Development for Exceptional Individuals, 30, 134-146.

Halpern, A. S., Herr, C. M., Wolf, N. K., Lawson, J. D, Doren, B., \& Johnson, M. D. (1995). NEXT S.T.E.P.: Student transition and educational planning. Teacher manual. Eugene, OR: University of Oregon.

Hasnain, R., \& Balcazar, F. (2009). Predicting community- versus facility-based employment for transition-aged young adults with disabilities: The role of race, ethnicity, and support systems. Journal of Vocational Rehabilitation, 31, 175-188.

Hughes, C., \& Carter, E. W. (2000). The transition handbook: Strategies high school teachers use that work! Baltimore, MD: Paul H. Brookes.

Hughes, C., Cosgriff, J., Agran, M., \& Washington, B. (2013). Student self-determination: A preliminary investigation of the role of participation in inclusive settings. Education and Training in Autism and Developmental Disabilities, 48, 3-17.

Individuals with Disabilities Education Act (IDEA) of 1990, PL 101-476, 20 U.S.C. ss1400 et seq.
Kalyanpur, M., \& Harry, B. (2012). Cultural reciprocity in special education: Building family-professional relationships. Baltimore, MD: Paul H. Brookes.

Kim, K.-H., \& Morningstar, M. E. (2005). Transition planning involving cultural and linguistically diverse families. Career Development for Exceptional Individuals, 28, 92-103.

Kohler, P. (1996). Taxonomy for transition programming: Linking research to practice. Champaign: University of Illinois at Urbana-Champaign, Transition Research Institute.

Landmark, L. J., Zhang, D. D., \& Montoya, L. (2007). Culturally diverse parents' experiences in their children's transition: Knowledge and involvement. Career Development for Exceptional Individuals, 30, 68-79.

Leake, D. W., Burgstahler, S., \& Izzo, M. V. (2011). Promoting transition success for culturally and linguistically diverse students with disabilities: The value of mentoring. Creative Education, 2, 121-129.

Martin, J. E., \& Marshall, L. H. (1995). ChoiceMaker: A comprehensive self-determination transition program. Intervention in School and Clinic, 30, 147-156.

Martin, J. E., Mithaug, D. E., Husch, J. V., Frazier, E. S., \& Huber Marshall, L. (2003). The effects of optimal opportunities and adjustments on job choices of adults with severe disabilities. In D.E. Mithaug, D. Mithaug, M. Agran, J. E. Martin, \& M. Wehmeyer (Eds.), Self-determined learning theory: Predictions, prescriptions, and practice (pp. 188-205). Mahwah, NJ: Erlbaum Associates.

National Longitudinal Transition Study-2 (2003). Youth with disabilities: A changing population. A report of findings from the national longitudinal transition study and the national longitudinal transition study-2. Retrieved from http://www.nlts2.org//reports/changepop_reports.html

National Research Council (2002). Minority students in special and gifted education. Washington, DC: National Academics Press.

National Secondary Transition Technical Assistance Center (2010). Evidence-based practices and predictors in secondary transition: What we know and what we still need to know. Charlotte, NC, NSTTAC. Retrieved from: http://nsttac.org/ sites/default/files/assets/pdf/pdf/ebps/ExecsummaryPPs $\% 20$ Jan2013.pdf

Raskind, M. H., Goldberg, R. J., Higgins, E. L., \& Herman, K. L. (1999). Patterns of change and predictors of success in individuals with learning disabilities: Results from a twenty-year longitudinal study. Learning Disabilities Research \& Practice, 14, 35-49.

Sanford, C., Newman, L., Wagner, M., Cameto, R., Knokey, A. M., \& Shaver, D. (2011). The post-high school outcomes of young adults with disabilities up to 6 years after high school: Key findings from the National Longitudinal Transition Study2 (NLTS2). NCSER 2011-3004. National Center for Special Education Research.

Shogren, K. A. (2011). Culture and self-determination: A synthesis of the literature and directions for future research and practice. Career Development for Exceptional Individuals, 34, 115-127.

Stodden, R. A., \& Dowrick, P. W. (2001). Postsecondary education and employment of adults with disabilities. American Rehabilitation, 25, 19-23.

Storey, K., \& Miner, C. (2011). Systematic instruction of functional skills for students and adults with disabilities. Springfield, IL: Charles C. Thomas. 
Thoma, C.A. (1999). Supporting student voice in transition planning. Teaching Exceptional Children, 30, 4-9.

Thoma, C. A., \& Getzel, E. E. (2005). Self-determination is what it's all about: What post-secondary student with disabilities tell us are important considerations for success. Education and Training in Mental Retardation and Developmental Disabilities, 40, 35-48.

Trainor, A. A. (2008). Using cultural and social capital to improve postsecondary outcomes and expand transition models for youth with disabilities. The Journal of Special Education, 42, 148-162.

U.S. Department of Education (2002). Twenty-fourth annual report to Congress on the implementation of the Individuals with Disabilities Education Act. Washington, DC: Author.

U.S. General Accounting Office (2003). Federal actions can assist states in improving postsecondary outcomes for youth. Washington, DC: Department of Education.

Wagner, M., Newman, L., Cameto, R., Garza, N., \& Levine, P. (2005). After high school: A first look at the postschool experiences of youth with disabilities. A report from the National Longitudinal Transition Study-2. Menlo Park, CA: SRI International.
Washington, B. H., Hughes, C., \& Cosgriff, J. C. (2012). High-poverty youth: Self-determination and involvement in educational planning. Career Development and Transition for Exceptional Individuals, 35, 14-28.

Wehman, P. (2011). Essentials of transition planning. Baltimore, MD: Paul H. Brookes.

Wehmeyer, M. L., Agran, M., \& Hughes, C. (1998). Teaching selfdetermination to students with disabilities. Baltimore, MD: Paul H. Brookes.

Wehmeyer, M. L., \& Sands, D. L. (Eds.). (1998). Making it happen: Student involvement in education planning, decision making, and instruction. Baltimore: Paul H. Brookes.

Wehmeyer, M. L. \& Schalock, R. (2001). Self-determination and quality of life: Implications for special education services and supports. Focus on Exceptional Children, 23, 1-16.

Wehmeyer, M. L., \& Schwartz, M. (1998). Self-determination and positive adult outcomes: A follow-up study of youth with mental retardation or learning disabilities. Exceptional Children, $63,245-255$. 